TRANSACTIONS OF THE

AMERICAN MATHEMATICAL SOCIETY

Volume 353 , Number 7 , Pages 2673-2688

S 0002-9947(01)02742-8

Article electronically published on January 18, 2001

\title{
INVARIANTS AND PROJECTIONS OF SIX LINES IN PROJECTIVE SPACE
}

\author{
DANA R. VAZZANA
}

\begin{abstract}
Given six lines in $\mathbf{P}^{3}$, quartics through the six lines define a map from $\mathbf{P}^{3}$ to $\mathbf{P}^{4}$, and the image of this map is described in terms of invariants of the six lines. The map can be interpreted as projection of the six lines, and this permits a description of the canonical model of the octic surface which is given by points which project the lines so that they are tangent to a conic. We also define polarity for sets of six lines, and discuss the above map in the case of a self-polar set of lines and in the case of six lines which form a "double-sixer" on a cubic surface.
\end{abstract}

\section{Invariants of Six Lines in Projective Space}

1.1. Invariants of Six Lines in $\mathbf{P}^{2}$. Consider a set of six lines in $\mathbf{P}^{2}$, where each line is described by the linear form $a_{i} x+b_{i} y+c_{i} z=0, i=1, \ldots, 6$. Using the coefficients $\left[a_{i}, b_{i}, c_{i}\right]$ of the linear forms which describe the lines, we can represent them by a $6 \times 3$ matrix $X$. We denote the $3 \times 3$ minor of $X$ given by the $i$ th, $j$ th, and $k$ th columns of $X$ by [ijk], and represent products of the $3 \times 3$ minors by tableaux:

$$
\left[\begin{array}{c}
i j k \\
l m n
\end{array}\right]=[i j k][l m n]
$$

Note that, via duality, the same construction can be used to describe points as well as lines. Following [DO], we describe the moduli space of 6 lines in $\mathbf{P}^{2}$ by homogeneous invariants of the set of lines, which are products of the matrices $[i j k]$ in which each index occurs an equal number of times. In [DO], it is shown that the moduli space $\mathbf{P}_{2}^{6}$ of six lines in $\mathbf{P}^{2}$ is defined by five degree one invariants and a single degree two invariant. The degree one invariants are listed below:

$$
t_{0}=\left[\begin{array}{l}
123 \\
456
\end{array}\right], \quad t_{1}=\left[\begin{array}{l}
124 \\
356
\end{array}\right], \quad t_{2}=\left[\begin{array}{l}
125 \\
346
\end{array}\right], \quad t_{3}=\left[\begin{array}{l}
134 \\
256
\end{array}\right], \quad t_{4}=\left[\begin{array}{l}
135 \\
246
\end{array}\right]
$$

Received by the editors July 25, 1999.

2000 Mathematics Subject Classification. Primary 14L24; Secondary 14Q10.

Key words and phrases. Algebraic geometry, invariant theory.

The author would like to thank Igor Dolgachev for his invaluable assistance in producing this research. 
The single degree two invariant needed to complete our description of the moduli space is $t_{5}=y_{1}-y_{2}$, where $y_{1}, y_{2}$ are as follows:

$$
y_{1}=\left[\begin{array}{c}
123 \\
145 \\
246 \\
356
\end{array}\right], \quad y_{2}=\left[\begin{array}{c}
124 \\
135 \\
236 \\
456
\end{array}\right] .
$$

We construct the moduli space $\mathbf{P}_{2}^{6}$ by considering $t_{0}, \ldots, t_{4}, t_{5}$ as coordinates on $\mathbf{P}(1,1,1,1,2)$. Straightening syzygies give the following relation between the six generators:

$$
\begin{aligned}
t_{5}^{2}= & \left(-t_{2} t_{3}+t_{1} t_{4}+t_{0} t_{1}+t_{0} t_{4}-t_{0} t_{2}-t_{0} t_{3}-t_{0}^{2}\right)^{2} \\
& -4 t_{0} t_{1} t_{4}\left(-t_{0}+t_{1}-t_{2}-t_{3}+t_{4}\right)
\end{aligned}
$$

The set of zeroes of the right-hand side of the above equation comprise an algebraic variety in $\mathbf{P}^{4}$ known as Igusa's quartic $I_{4}$. The moduli space $\mathbf{P}_{2}^{6}$ then embeds in $\mathbf{P}(1,1,1,1,2)$ as the solution of the equation $t_{5}^{2}=I_{4}$, where $I_{4}$ is Igusa's quartic.

Consider the equation $y_{1}-y_{2}=0$. The significance of this locus in the moduli space is most easily seen by considering sets of points instead of lines. By fixing five points and letting the sixth point vary, we see that $y_{1}-y_{2}$ represents a curve of degree two which vanishes on all six points. Therefore, we can see that Igusa's quartic parametrizes sets of six points that lie on a conic in $\mathbf{P}^{2}$, or dually, sets of six lines that are tangent to a conic.

Note that only semistable (see [DO]) sets of lines are represented by points in the moduli space constructed above. It is shown in [DO] that configurations with one double line are allowed, as is a configuration in which four lines intersect in a common point. A configuration in which five lines intersect in a common point is not semistable, and will not correspond to a point in the moduli space.

1.2. Association for Six Lines in $\mathbf{P}^{2}$. We now wish to discuss the concept of association, which was known classically for sets of six lines in $\mathbf{P}^{2}$. Using the coordinates $\left\{t_{0}, t_{1}, t_{2}, t_{3}, t_{4}\right\}$, we can represent the moduli space $\mathbf{P}_{2}^{6}$ as a double cover of $\mathbf{P}^{4}$, branched along Igusa's quartic. It is shown in [DO that pairs of points in $\mathbf{P}_{2}^{6}$ which map to the same point in $\mathbf{P}^{4}$ correspond to configurations of lines in $\mathbf{P}^{2}$ which are associated, where association is defined for two sets of six lines in $\mathbf{P}^{2}$ as follows: $\left\{l_{1}, \ldots, l_{6}\right\},\left\{l_{1}^{\prime}, \ldots, l_{6}^{\prime}\right\}$ are associated if their respective coordinate matrices $X, Y$ satisfy $X \cdot \Lambda \cdot Y^{T}=0$ for some diagonal matrix $\Lambda$ such that $\operatorname{det}[\Lambda]$ is nonzero.

In [ $[\mathrm{Ht}]$, B. Hunt gives an equivalent definition of association for six lines in $\mathbf{P}^{2}$ (due to G. Castelnuovo) which is more geometric in nature:

Definition 1.1. $\left\{l_{1}, \ldots, l_{6}\right\},\left\{l_{1}^{\prime}, \ldots, l_{6}^{\prime}\right\}$ are associated if the following relation holds. Since the set $\left\{l_{1}, \ldots, l_{6}\right\}$ is ordered, we can form two triangles

$$
\Delta\left(l_{1}, l_{2}, l_{3}\right), \quad \Delta\left(l_{4}, l_{5}, l_{6}\right) ;
$$

and these two triangles have together six vertices, which come equipped with a numbering, say $\left(p_{1}, \ldots, p_{6}\right)$, and these correspond dually (with respect to a conic) to another ordered set of six lines, $\left(l_{p_{1}}, \ldots, l_{p_{6}}\right)$. Then $\left\{l_{1}, \ldots, l_{6}\right\},\left\{l_{1}^{\prime}, \ldots, l_{6}^{\prime}\right\}$ are associated if $\left(l_{p_{1}}, \ldots, l_{p_{6}}\right)=\left\{l_{1}^{\prime}, \ldots, l_{6}^{\prime}\right\}$ as a set of six ordered lines.

It is easy to see that the definition does not depend on the conic used. Recall that sets of six lines which correspond to points on the branch divisor $I_{4}=0$ are 
necessarily tangent to a conic in $\mathbf{P}^{2}$. We define such configurations to be selfassociated.

1.3. Invariants of Lines in $\mathbf{P}^{3}$. Consider a set of lines $l_{1}, \ldots, l_{n}$ in $\mathbf{P}^{3}$. Each line $l_{i}$ can be given by two points $u_{i}=\left(u_{0}^{i}, u_{1}^{i}, u_{2}^{i}, u_{3}^{i}\right), v_{i}=\left(v_{0}^{i}, v_{1}^{i}, v_{2}^{i}, v_{3}^{i}\right)$. We can then form a basic invariant $\left[l_{i}, l_{j}\right]$ as follows:

$$
\left[l_{i}, l_{j}\right]=\operatorname{det}\left[\begin{array}{cccc}
u_{0}^{i} & u_{1}^{i} & u_{2}^{i} & u_{3}^{i} \\
v_{0}^{i} & v_{1}^{i} & v_{2}^{i} & v_{3}^{i} \\
u_{0}^{j} & u_{1}^{j} & u_{2}^{j} & u_{3}^{j} \\
v_{0}^{j} & v_{1}^{j} & v_{2}^{j} & v_{3}^{j}
\end{array}\right]
$$

(See [Sf], Section 3.4, for a more complete discussion of these invariants.)

For convenience, we will sometimes denote the invariant $\left[l_{i}, l_{j}\right]$ by simply $[i j]$. We can construct the invariant $[i j]$ as an inner product of the Plücker coordinates of $l_{i}$ and $l_{j}$. Let $\overline{l_{i}}=\left(l_{12}^{i}, l_{13}^{i}, l_{14}^{i}, l_{23}^{i}, l_{24}^{i}, l_{34}^{i}\right)$ denote the vector of Plücker coordinates for $l_{i}$. The invariant $\left[l_{i}, l_{j}\right]$ is then given as follows:

$$
\left[l_{i}, l_{j}\right]=l_{12}^{i} l_{34}^{j}-l_{13}^{i} l_{24}^{j}+l_{14}^{i} l_{23}^{j}+l_{23}^{i} l_{14}^{j}-l_{24}^{i} l_{13}^{j}+l_{34}^{i} l_{12}^{j} .
$$

From these, we form the following degree one homogeneous invariants:

$$
\begin{aligned}
q_{0} & =[12][34][56] \\
q_{1} & =[12][35][46] \\
q_{2} & =[12][36][45] \\
q_{3} & =[13][24][56] \\
q_{4} & =[13][25][46] \\
q_{5} & =[13][26][45] \\
q_{6} & =[14][23][56] \\
q_{7} & =[14][25][36] \\
q_{8} & =[14][26][35] \\
q_{9} & =[15][23][46] \\
q_{10} & =[15][24][36] \\
q_{11} & =[15][26][34] \\
q_{12} & =[16][23][45] \\
q_{13} & =[16][24][35] \\
q_{14} & =[16][25][34]
\end{aligned}
$$

We can form another homogeneous invariant by taking the determinant of the matrix $M$ whose $i^{t h}$ row consists of the Plücker coordinates for the line $l_{i}$. We will denote this additional invariant by $D$. It can be shown that the moduli space of sets of six lines in $\mathbf{P}^{3}$ is realized as a double cover of a toric variety generated by the invariants $q_{i}$, branched over the locus $D=0$. This is the subject of a paper which is currently in preparation.

With respect to the above quadratic form, the dual Plücker coordinates of a line $l$ are given as follows:

$$
\overline{l_{i}^{\prime}}=\left(l_{34}^{i},-l_{24}^{i}, l_{23}^{i}, l_{14}^{i},-l_{13}^{i}, l_{12}^{i}\right) .
$$


By taking the product of the matrix $M$ with the matrix $M^{\prime}$ formed by the dual lines $\overline{l_{i}^{\prime}}$, we can see that the matrices $M, M^{\prime}$ satisfy the following relation:

$$
M M^{\prime T}=\left[\begin{array}{cccccc}
0 & {[12]} & {[13]} & {[14]} & {[15]} & {[16]} \\
{[12]} & 0 & {[23]} & {[24]} & {[25]} & {[26]} \\
{[13]} & {[23]} & 0 & {[34]} & {[35]} & {[36]} \\
{[14]} & {[24]} & {[34]} & 0 & {[45]} & {[46]} \\
{[15]} & {[25]} & {[35]} & {[45]} & 0 & {[56]}
\end{array}\right]
$$

We will denote the above matrix by $A$. We can see that the matrix $M^{\prime}$ can be obtained from $M$ by interchanging the first and last columns, second and fifth columns, and third and fourth columns, as well as changing the sign of the second and fifth columns. Therefore, $\operatorname{det} M=-\operatorname{det} M^{\prime}$, and

$$
-D^{2}=\operatorname{det} A,
$$

where $\operatorname{det} A$ can be expressed as a polynomial in the (inhomogeneous) invariants $[i j]$.

1.4. Polarity for Six Lines in $\mathbf{P}^{3}$. We now wish to define polarity for sets of six lines in $\mathbf{P}^{3}$, in a manner analogous to association for lines in $\mathbf{P}^{2}$. We begin by letting each line $l_{i}$ in $\mathbf{P}^{3}$ be defined by two points $u_{i}, v_{i}$ in $\mathbf{P}^{3}$. Let $x_{i}$ be the $2 \times 4$ matrix whose columns are $u_{i}, v_{i}$. Then $x_{i}, x_{i}^{\prime}$ are dual with respect to the quadric $Q=\alpha_{0}^{2}+\alpha_{1}^{2}+\alpha_{2}^{2}+\alpha_{4}^{2}$ if and only if the following relation holds:

$$
x_{i}^{T} x_{i}^{\prime}=0 .
$$

(Equivalently, $\bar{s} \cdot \bar{t}$ for all $\bar{s} \in l_{i}, \bar{t} \in l_{i}^{\prime}$.) Notice that, using this quadric, the dual line is the same as that given by the dual Plücker coordinates. We now need the following theorem.

Theorem 1.2. The dual set of six lines described up to projective equivalence is independent of the quadric chosen.

Note that the quadric $Q$ defines a quadratic form which is represented by the identity matrix. Let $Q^{\prime}$ be any other nonsingular quadric in $\mathbf{P}^{3}$. Then, $Q^{\prime}=S Q$, where $S \in G L_{4}(\mathbf{C})$.

The quadratic form represented by $Q^{\prime}$ can be given by the matrix

$$
S^{T} S
$$

since $Q$ is given by the identity matrix. We want to show that the dual lines defined by the quadric $Q^{\prime}$ are projectively equivalent to the dual lines defined by the quadric $Q$.

As $S \in G L_{4}(\mathbf{C})$, we can see that the set of lines defined by the matrices $\left\{x_{1}^{\prime}, \ldots, x_{6}^{\prime}\right\}$ are projectively equivalent to the lines defined by the matrices $\left\{\left(S^{T} S\right)^{-1} x_{1}^{\prime}, \ldots,\left(S^{T} S\right)^{-1} x_{6}^{\prime}\right\}$. However,

$$
x_{i}^{T} S^{T} S\left(S^{T} S\right)^{-1} x_{i}^{\prime}=x_{i}^{T} x_{i}^{\prime}=0
$$

for all $i$, and since the dual lines thus defined are unique, we see that the duality is independent of the quadric chosen.

Definition 1.3. Given a set of six lines in $\mathbf{P}^{3}$ described up to projective equivalence, the polar set of lines to the given lines is the set of dual lines with respect to any nonsingular quadric, given up to projective equivalence. 
We can see that the polar set of lines will have the same invariants $[i j]$, but the invariant $D^{\prime}$ of the polar set of lines will equal $-D$.

The phenomenon of polarity for sextuples of lines in $\mathbf{P}^{3}$ is especially interesting when we consider the special case of six lines which lie on a nonsingular cubic surface. In this case, any six nonintersecting lines form a double-sixer, which is a configuration in which each subset of five out of the six lines has a common transversal.

It was proved by Schur $[\mathrm{Sc}]$ that for any double-sixer there exists a quadric, known now as the Schur quadric, with respect to which the dual of any line is the transversal of the remaining five lines. Therefore, the polar set of lines under our previous definition is consistent up to projective equivalence with the previously known duality for the two sextuples of lines in any double-sixer.

1.5. Self-Polarity and the Linear Complex. We now wish to consider the case in which the set $l_{i}$ of lines is projectively equivalent to the set $l_{i}^{\prime}$ of polar, in which case we will define the set of lines to be self-polar. Recalling that $D^{\prime}=-D$ in this case, we observe that $D=0$ for a set of self-polar lines, and that therefore the six lines must belong to a linear complex, in which the six lines are linearly dependent as points in the Plücker space. The following theorem, due to I. Dolgachev, uses Chasles's theorem ([Stu, p. 102) to confirm this.

Theorem 1.4. Let $\ell=\left(l_{1}, \ldots, l_{6}\right)$ be an ordered set of six lines in $P^{3}$. For any non-degenerate quadric $Q$ we denote by $\ell_{Q}{ }^{*}$ the set of polar lines with respect to $Q$. The following properties are equivalent:

(i) $\ell_{Q}{ }^{*}$ is projectively equivalent to $\ell$ for some $Q$;

(ii) $\ell_{Q}{ }^{*}$ is projectively equivalent to $\ell$ for any $Q$;

(iii) the set $\ell$ belongs to a linear complex of lines (i.e. the six lines are linearly dependent as points in the Plucker space).

Proof. The equivalence of $(i)$ and $(i i)$ follows directly from Theorem 1.2. To prove $($ ii $) \Rightarrow($ iii $)$ we use the following:

Theorem 1.5. (Chasles, 1849). Let $Q$ be a non-degenerate quadric in $P^{3}$ and $\sigma$ be an involution of $Q$ such that it is identical on one of the rulings $\pi_{1}: Q \rightarrow P^{1}$ (i.e. $\pi_{1} \circ \sigma=\pi_{1}$ ). Then the set of lines which are either lines from the ruling $\pi_{1}$ or intersect $Q$ at the lines $l, l^{\prime}$ from the second ruling $\pi_{2}: Q \rightarrow P^{1}$ which are in the involution $\sigma$ (i.e. $\left.\sigma(l)=l^{\prime}\right)$ form a linear complex of lines. Conversely each linear complex of lines can be obtained in such a way.

In order to prove Chasles's theorem, we first need the following lemma:

Lemma 1.6. Let $m \in K$ intersect a line $l$ (not necessarily in $K$ ), then $m$ intersects its polar line $\check{l}$. The complex $K$ consists of lines intersecting some pair of polar lines.

Proof. Let $p=l \cap m$. The Nullplane $\pi(p)$ of $p$ contains $\check{l}$. It also contains $m$ (since $\check{m}=m$ ). Thus $m$ intersects $\check{l}$. Suppose $m$ intersects a pair of polar lines $l$ and $\check{l}$ at the points $x$ and $x^{\prime}$, respectively. Then the Nullplane $\Pi(x)$ contains $\check{l}$ and hence $x^{\prime}$. It must also contain its Nullpoint $x$. The Nullplane $\Pi\left(x^{\prime}\right)$ contains $l$ and hence $x$ and $x^{\prime}$. Thus $\check{m}=\Pi(x) \cap \Pi\left(x^{\prime}\right)$ contains both $x$ and $x^{\prime}$, hence coincides with itself. Therefore $m \in K$. 
Proof of Chasles's Theorem. Let $K$ be a linear complex and $\left(l, l^{\prime}\right),\left(m, m^{\prime}\right)$ be two pairs of polar lines with respect to $K$, none of which intersect. Consider a line $\lambda \in K$ which intersects $(l, m)$. By Lemma 1.6, $\lambda$ intersects $l^{\prime}, m^{\prime}$. Thus, any line from $K$ which intersects $l, m$ intersects $l, l^{\prime}, m, m^{\prime}$. So, if we consider the quadric $Q$ formed by $l, l^{\prime}, m$, each line $\lambda \subset K$ intersecting $l, m$ belongs to $Q$ and also intersects $m^{\prime}$. This shows that $m^{\prime} \subset Q$ and we have two rulings in $Q$. The first one consists of lines from $K$, the second one contains two pairs of polar lines $\left(l, l^{\prime}\right),\left(m, m^{\prime}\right)$. For any line $s$ from the second ruling, its polar line $\check{s}$ must belong to the same ruling (because by Lemma 2.6, it intersects all lines from the first ruling). Thus the polarity defines an involution on the second ruling, and hence defines an involution $\sigma$ on $Q$ satisfying the condition of the theorem. Each line from $K$ not from the first ruling intersects $Q$ at two points, each belonging to a line from the first ruling. By Lemma 1.6, these two lines form a polar pair. Thus, $K$ consists of lines defined by $(Q, \sigma)$ as in the theorem.

Conversely, let $K$ be the set of lines defined by $(Q, \sigma)$ as in Chasles's theorem. As all quadrics are projectively equivalent, it suffices to show that the the lines defined by $(Q, \sigma)$ for a particular quadric $Q$ form a linear complex. Let $Q$ be defined by the equation $x_{0} x_{1}-x_{2} x_{3}=0$. There are two rulings on $Q$. One consists of lines of the form $x_{0}=\alpha x_{2}, x_{3}=\alpha x_{1}$, and the other consists of lines of the form $x_{0}=\beta x_{3}, x_{2}=\beta x_{1}$. Denote the two rulings by $\pi_{1}, \pi_{2}$, respectively.

There are then two possibilities for the involution $\sigma$. If the involution $\sigma$ preserves the lines from $\pi_{1}$, then $\sigma$ must take the following form for some $a, b, c \in \mathbf{C}$ :

$$
\left[\begin{array}{cccc}
a & 0 & 0 & b \\
0 & -a & c & 0 \\
0 & b & a & 0 \\
c & 0 & 0 & -a
\end{array}\right] .
$$

If $\sigma$ preserves the lines from the second ruling $\pi_{2}$, then $\sigma$ takes this form:

$$
\left[\begin{array}{cccc}
a & 0 & b & 0 \\
0 & -a & 0 & c \\
c & 0 & -a & 0 \\
0 & b & 0 & a
\end{array}\right] .
$$

The proof is similar in both cases, so we will give details only for the first ruling.

Suppose a line $m$ meets lines $l, l^{\prime}$ which are in the second ruling. Let $x=m \cap l$. Then $x$ must be of the form

$$
(\alpha, s, \alpha s, 1) \text {. }
$$

The involution $\sigma$ transforms the line $l$ to one of the form

$$
(a \alpha+b,-a t+c \alpha t, b t+a \alpha t, \alpha c-a) .
$$

Therefore, the line $m$ can be determined by two points, one of the form $(\alpha, s, \alpha s, 1)$ and one of the form $(a \alpha+b,-a t+c \alpha t, b t+a \alpha t, \alpha c-a)$ for some values of $s, t$.

However, it can be checked that any such line satisfies the linear equation

$$
a x_{01}-c x_{02}-b x_{13}-a x_{23}=0
$$

in the Plücker space, and thus belongs to a linear complex. All lines from the first ruling have Plücker coordinates of the form

$$
\left(\alpha, 0, \alpha^{2},-1,0, \alpha\right)
$$


and therefore also satisfy the above linear equation. Thus, the equation defines the linear complex $K$, and the proof is complete.

To deduce $(i i) \Rightarrow$ (iii) from Chasles's theorem we choose $Q$ to be the quadric spanned by $l_{1}, l_{2}, l_{2}$. We identify $Q$ with $P^{1} \times P^{1}$ by means of its two rulings, the first factor corresponds to the ruling containing the lines $l_{1}, l_{2}, l_{3}$. Then $\ell_{Q}{ }^{*}=$ $\left(l_{1}, l_{2}, l_{3}, l_{4}^{\prime}, l_{5}^{\prime}, l_{6}^{\prime}\right)$. Moreover, if $l_{i}$ intersects $Q$ at the points $\left(a_{i}, b_{i}\right),\left(a_{i}^{\prime}, b_{i}^{\prime}\right), i=$ $4,5,6$, then $l_{i}^{\prime}$ intersects $Q$ at the points $\left(a_{i}, b_{i}^{\prime}\right),\left(a_{i}^{\prime}, b_{i}\right)$. Suppose $\ell_{Q}{ }^{*}=A \ell$ for some linear transformation $A$. Since $A$ fixes $l_{1}, l_{2}, l_{3}$, it preserves the first ruling. Since $A l_{i}=l_{i}^{\prime}, i=4,5,6$, it acts on the second ruling by switching $\left(b_{i}, b_{i}^{\prime}\right) \leftrightarrow\left(b_{i}^{\prime}, b_{i}\right)$. This immediately implies that $A$ defines an involution on $Q$ which satisfies Chasles's theorem. Hence the lines $l_{1}, l_{2}, l_{3}, l_{4}, l_{5}, l_{6}$ (as well as $\left.\left(l_{1}, l_{2}, l_{3}, l_{4}^{\prime}, l_{5}^{\prime}, l_{6}^{\prime}\right)\right)$ belong to a linear complex $K$.

Now let us deduce $($ iii $) \Rightarrow($ i $)$ from Chasles's theorem. Each non-degenerate linear complex $K$ defines the polarity $l \rightarrow \check{l}$ with respect to $K$. We view $K$ as an element of $\Lambda^{2}\left(V^{*}\right)$ or as a bijective linear map $u_{K}: V \rightarrow V^{*}$. This map satisfies $u_{K}(v)(v)=K(v, v)=0$ for all $v \in V$. The plane $\Pi(x): u_{k}(v)=0$ is the Nullplane of the point $x=[v] \in P(V)$. It contains $x$. The point $x$ is the Nullpoint of the plane $\pi$. The intersection of all Nullplanes of points on a line $l$ is the polar line $\check{l}$. Clearly,

$$
l \in K \Longleftrightarrow l=\check{l} .
$$

Let $l, l^{\prime}$ be two transversals of $l_{1}, l_{2}, l_{3}, l_{4}$. By the lemma, the polar line $\check{l}$ intersects $l_{1}, l_{2}, l_{3}, l_{4}$, hence must coincide either with $l$ or $l^{\prime}$. The first case is impossible. In fact, if $l=\check{l}$, then $l \in K$. Then the pencil of lines through $l \cap l_{1}$ in the plane $\left\langle l, l_{1}\right\rangle$ is contained in $K$. Similarly, the pencil of lines through $l \cap l_{2}$ in the plane $\left\langle l, l_{2}\right\rangle$ is contained in $K$. But these two pencils span a plane in $P^{5}$ which cuts out the union of two lines in $G(2,4)$. Since $K$ is non-degenerate it cuts on each plane a non-degenerate conic in $G(2,4)$. Contradiction.

Thus we see that the pair of transversals is a pair of polar lines, i.e. $l^{\prime}=\check{l}$. Now the pair of transversals $m, m^{\prime}$ to $l_{1}, l_{2}, l_{3}, l_{5}$ is also a pair of polar lines. Consider the quadric $Q$ spanned by $l_{1}, l_{2}, l_{3}$. The four transversals $l, l^{\prime}, m, m^{\prime}$ are the four lines from the second ruling of $Q$ (the first one contains $\left.l_{1}, l_{2}, l_{3}\right)$ which form two pairs of polar lines. We can always find an involution $\sigma$ on $Q$ which preserves the first ruling and $\sigma(l)=l^{\prime}, \sigma(m)=m^{\prime}$. Consider the linear complex $K^{\prime}$ defined by $(Q, \sigma)$. Since $l_{1}, l_{2}, l_{3}, l_{4}, l_{5}$ belong to $K$, and any linear complex is determined by 5 linear independent lines (here we have to assume that the first 5 lines are Plücker independent), we have the equality $K=K^{\prime}$. Thus $l_{6}$ intersects $Q$ at a pair of the lines in the second ruling which are in the involution. This implies that $\ell_{Q}{ }^{*}=\sigma(\ell)$, hence (i). End of the proof.

\section{Projections of Six Lines in $\mathbf{P}^{3}$}

2.1. Hierhölzer's Octic Surface. We now turn to the case of projection of six lines in $\mathbf{P}^{3}$. Given six lines in $\mathbf{P}^{3}$, we will define general position for the six lines as follows:

1. No two of the lines intersect

2. No subset of five out of the six lines has a common transversal.

In particular, note that the six lines of a "double-sixer" on a cubic surface are not in general position. We will treat this case separately. 
In his 1870 paper Ueber Kegelschnitte im Raume [Hz, Hierhölzer first introduces an octic surface $O_{8}$ with the following property: given six lines $\left\{l_{1}, l_{2}, l_{3}, l_{4}, l_{5}, l_{6}\right\}$ in $\mathbf{P}^{3}, O_{8}$ is the closure of the set of points $p$ in $\mathbf{P}^{3}$ such that projection from $p$ yields six lines which are tangent to a conic. Recently, Bruce Hunt has drawn some nice pictures of the Hierhölzer surface for his Gallery of Algebraic Surfaces (see Ht2]). We will show how to use the previous discussion of moduli spaces in order to describe the canonical model of the Hierhölzer surface.

Let each line $l_{i}$ be given by points $v_{i}=\left(\alpha_{i}, \beta_{i}, \gamma_{i}, \delta_{i}\right), w_{i}=\left(\alpha_{i}^{\prime}, \beta_{i}^{\prime}, \gamma_{i}^{\prime}, \delta_{i}^{\prime}\right)$, for $i=1, \ldots, 6$. Denote the point of projection by $p=(a, b, c, d)$, and choose the fixed plane to be the plane $d=0$ in $\mathbf{P}^{3}$ with coordinates $(a, b, c, d)$. Without loss of generality, we can let $\delta_{i}=0$, and we can assume that no line $l_{i}$ is contained in the plane $d=0$. The coordinates of the image line $\operatorname{proj}_{p}\left(l_{i}\right)$ are then given as follows:

$$
\begin{aligned}
& \operatorname{proj}_{p}\left(v_{i}\right)=\left(\alpha_{i}, \beta_{i}, \gamma_{i}\right), \\
& \operatorname{proj}_{p}\left(w_{i}\right)=\left(d \alpha_{i}^{\prime}-a \delta_{i}^{\prime}, d \beta_{i}^{\prime}-b \delta_{i}^{\prime}, d \gamma_{i}^{\prime}-c \delta_{i}^{\prime}\right) .
\end{aligned}
$$

Taking the wedge product, we get

$$
\operatorname{proj}_{p}\left(l_{i}\right)=\left(x_{i}(p), y_{i}(p), z_{i}(p)\right),
$$

where

$$
\begin{aligned}
& x_{i}=\left(\alpha_{i} \beta_{i}^{\prime}-\alpha_{i}^{\prime} \beta_{i}\right) d-\alpha_{i} \delta_{i}^{\prime} b+\beta_{i} \delta_{i}^{\prime} a, \\
& y_{i}=\left(\alpha_{i} \gamma_{i}^{\prime}-\alpha_{i}^{\prime} \gamma_{i}\right) d-\alpha_{i} \delta_{i}^{\prime} c+\gamma_{i} \delta_{i}^{\prime} a, \\
& z_{i}=\left(\beta_{i} \gamma_{i}^{\prime}-\beta_{i}^{\prime} \gamma_{i}\right) d-\beta_{i} \delta_{i}^{\prime} c+\gamma_{i} \delta_{i}^{\prime} b .
\end{aligned}
$$

We now have expressed the coordinates of the image lines as linear functions of the point of projection. From these, we can form the following $6 \times 3$ matrix:

$$
\left[\begin{array}{lll}
x_{1} & y_{1} & z_{1} \\
x_{2} & y_{2} & z_{2} \\
x_{3} & y_{3} & z_{3} \\
x_{4} & y_{4} & z_{4} \\
x_{5} & y_{5} & z_{5} \\
x_{6} & y_{6} & z_{6}
\end{array}\right] .
$$

Consider the minor $[i j k]$. At first glance, the minors are cubic polynomials in $\mathbf{P}^{3}$, with coordinates $(a, b, c, d)$. However, each minor factors as $d \cdot q_{i j k}$, where $q_{i j k}$ can be viewed as the quadric surface which is determined by the three skew lines $l_{i}, l_{j}, l_{k}$. By the discussion in Section 1.1, we see that Hierhölzer's octic $O_{8}$ is given by the vanishing of the polynomial

$$
h=q_{123} q_{145} q_{246} q_{356}-q_{124} q_{135} q_{236} q_{456} .
$$

As observed in $\mathrm{Ca}, O_{8}$ has as singularities only the lines $\left\{l_{1}, l_{2}, l_{3}, l_{4}, l_{5}, l_{6}\right\}$, which are ordinary double lines. Also, the 30 transversals which intersect subsets of four out of the six lines lie on the surface $O_{8}$. We now wish to observe the behavior of the surface $O_{8}$ under the canonical map. The canonical divisor is given here by the linear system of quartics through the six lines. Since the dimension of the space of quartics in $\mathbf{P}^{3}$ is $\left(\begin{array}{l}7 \\ 4\end{array}\right)-1=34$ and each line imposes 5 extra linear conditions, we can see that the canonical model of $O_{8}$ will map into $\mathbf{P}^{4}$. With this information, we can now describe the surface invariants of $O_{8}$.

Theorem 2.1. The canonical model of Hierhölzer's octic surface embeds in $\mathbf{P}^{4}$ with 30 nodes, and has invariants $p_{g}=5$ and $K^{2}=8$. 


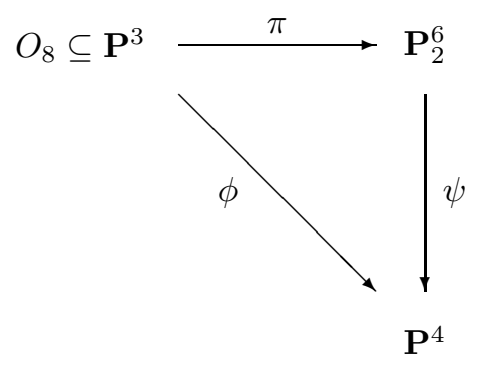

FiguRE 1.

The 30 transversals of the subsets of 4 out of the 6 lines can be seen by Bezout's Theorem to blow down to the 30 nodes of the surface $O_{8}$. The linear system of quartics through the six lines has vector space dimension five, as above, and therefore $p_{g}=5$. Finally, the calculation $K^{2}=8$ follows directly from the formulae in $\mathrm{GH}$, p. 624 .

A convenient choice of quartics for the canonical map $\phi$ is as follows:

$$
\begin{aligned}
t_{0} & =q_{123} q_{456}, \\
t_{1} & =q_{124} q_{356}, \\
t_{2} & =q_{125} q_{346}, \\
t_{3} & =q_{134} q_{256}, \\
t_{4} & =q_{135} q_{246} .
\end{aligned}
$$

Note the correspondence here between the quartics chosen and the invariants for six lines in $\mathbf{P}^{2}$. The functions $t_{i}$ define a map from $\mathbf{P}^{3}$ to the degree one invariants of the image sets of lines which can be interpreted as projection from a point in $\mathbf{P}^{3}$. We have the following commutative diagram: The image of $\mathbf{P}^{3}$ under $\phi$ is given by a quartic $K_{4}$ in $\mathbf{P}^{4}$, as follows: let $K$ denote $\phi\left(\mathbf{P}^{3}\right)$. Take

$$
h_{1} \cdot h_{2} \cdot h_{3} \cdot K
$$

to be the intersection of arbitrary hyperplanes $h_{1}, h_{2}, h_{3}$ in $\mathbf{P}^{4}$ with $K$. This will give the degree of $K$.

Consider the preimages of the three hyperplanes under $\phi$. They are quartic surfaces $Q_{1}, Q_{2}, Q_{3}$ in $P^{3}$ through the six lines. We want to compute the intersection multiplicity of the quartics outside of the six lines.

We will regard $Q_{2}, Q_{3}$ as divisors on $Q_{1}$. We have

$$
\begin{aligned}
& Q_{1} \sim l_{1}+l_{2}+l_{3}+l_{3}+l_{4}+l_{5}+l_{6}+C_{1}, \\
& Q_{2} \sim l_{1}+l_{2}+l_{3}+l_{3}+l_{4}+l_{5}+l_{6}+C_{2},
\end{aligned}
$$

where $C_{1}, C_{2}$ are the residual curves of degree ten.

We want to find $C_{1} \cdot C_{2}$. Notice that, for any line $l_{i}$, we have

$$
4=Q_{1} \cdot l_{i}=l_{i} \cdot l_{i}+l_{i} \cdot C_{1},
$$

and since $l_{i}^{2}=-2$, we see that $l_{i} \cdot C_{1}=6$.

Intersection of $C_{1}$ with $Q_{2}$ yields

$$
40=C_{1} \cdot Q_{2}=36+C_{1} \cdot C_{2},
$$


and thus the residual intersection of the curve is equal to four. Thus, the degree $d$ of $K$ can be no more than four. To verify that the degree of $K$ is no less than four in general, we refer to [T] (pp. 53-54).

It is clear that the image of $O_{8}$ under $\phi$ is contained in Igusa's quartic $I_{4}$. We will prove in the next section that $K_{4}$ and $I_{4}$ are tangent along a quadric $Q$, and that the canonical model of $O_{8}$ is given by the intersection of $I_{4}$ and $Q$.

We now wish to discuss the configuration of singularities on $O_{8}$. Cayley discusses interesting structures on the surface $O_{8}$, as follows:

1. the 30 transversals $t_{i j k l}, t_{i j k l}^{\prime}$,

2. the ten quartic curves $Q_{i j k}$,

given by the intersections of quadrics $q_{i j k}$ through $l_{i}, l_{j}, l_{k}$ with the quadrics $q_{l m n}$ through the complementary lines $l_{l}, l_{m}, l_{n}$.

We have already determined that the transversals blow down to 30 singular points, or nodes, on the canonical model of $O_{8}$. These are given by the intersection of the quadric $Q$ with the 15 singular lines of the Igusa quartic. Now, consider the ten quartic curves $Q_{i j k}$. It is easy to see, by inspection of the equation for $O_{8}$, that all such curves lie on Hierhölzer's surface. Each curve $Q_{i j k}=q_{i j k} \cap$ $q_{l m n}$ must pass through the twelve nodes given by the six pairs of transversals $t_{i j k l}, t_{i j k m}, t_{i j k n}, t_{i l m n}, t_{j l m n}, t_{k l m n}$. We would expect, then, that each node will lie on $\frac{12 \times 10}{30}=4$ quartic curves.

Consider the following syzygies on $3 \times 2$ tableaux:

$$
\begin{aligned}
& {[126][345]=[123][456]-[124][356]+[125][346]} \\
& {[136][245]=[135][246]-[123][456]-[134][356]} \\
& {[146][235]=[135][246]-[123][456]-[125][346]} \\
& {[156][234]=[134][256]+[123][456]-[124][356]} \\
& {[145][236]=[124][356]+[135][246]-[123][456]-[125][346]-[134][256]}
\end{aligned}
$$

As these correspond to relations between the quadric surfaces $q_{i} j k$, we can quickly see that each curve $Q_{i j k}$ is contained in a hyperplane section of Hierholzer's surface. In fact, along each such hyperplane section the Igusa quartic $I_{4}$ factors as the square of a quadric. Therefore, the images of the curves $Q_{i j k}$ under the canonical map are still given by quartic curves. It can be checked that each pair of nodes (given by a pair of transversals) lies in four such hyperplane sections, and therefore on four quartic curves as expected.

2.2. The Invariant Equation of $O_{8}$. Our research here shows that both $K_{4}$ and $Q$ can be expressed in terms of the invariants $\left\{q_{0}, \ldots, q_{14}\right\}$ and $D$ described in Section 1.3. In order to describe the situation completely, we need the following theorem.

Theorem 2.2. The coefficients of the quadric $Q$ can be expressed as linear functions of the invariants $\left\{q_{0}=(12)(34)(56), \ldots, q_{14}=(16)(25)(34)\right\}$. Let $Q$ be given by the equation

$$
Q=c_{0} t_{0}^{2}+\cdots+c_{4} t_{4}^{2}+c_{01} t_{0} t_{1}+c_{02} t_{0} t_{2}+\cdots+c_{34} t_{3} t_{4} .
$$


Then, the coefficients of $Q$ are given as follows:

$$
\begin{aligned}
c_{0} & =q_{0}+q_{1}-q_{2}+q_{3}+q_{4}-q_{5}-q_{6}+q_{7}+q_{8}-q_{9}+q_{10}+q_{11}+q_{12}-q_{13}-q_{14} \\
c_{1} & =2 q_{4} \\
c_{2} & =2 q_{3} \\
c_{3} & =2 q_{1} \\
c_{4} & =2 q_{0} \\
c_{01} & =2 q_{11}-2 q_{4}-c_{0} \\
c_{02} & =c_{0}+2 q_{3}-2 q_{8} \\
c_{03} & =c_{0}+2 q_{1}-2 q_{10} \\
c_{04} & =2 q_{7}-2 q_{0}-c_{0} \\
c_{12} & =2 q_{5}-2 q_{4}-2 q_{3} \\
c_{13} & =2 q_{9}-2 q_{1}-2 q_{4} \\
c_{14} & =c_{0}+2 q_{14}-2 q_{7}-2 q_{11} \\
c_{23} & =c_{0}+2 q_{13}-2 q_{10}-2 q_{8} \\
c_{24} & =2 q_{6}-2 q_{3}-2 q_{0} \\
c_{34} & =2 q_{2}-2 q_{0}-2 q_{1} \\
&
\end{aligned}
$$

Finally, $Q$ satisfies the relation

$$
\phi^{*}(Q)-D h=0,
$$

where the polynomials $t_{i}$ are lifted to $\mathbf{P}^{3}$ via $\phi$, and $h$ is the polynomial expression given at the beginning of the chapter for Hierhölzer's surface in $\mathbf{P}^{3}$.

The initial conjecture giving the coefficients of $Q$ rests on the fact that all 30 transversals are contained in Hierhölzer's surface. Each transversal intersects the quartics $t_{i}$ in four points; therefore, Bezout's Theorem shows us that the map $\phi$ blows down each transversal to a point on the canonical model. We can describe the coordinates of these points by the following lemma:

Lemma 2.3. On the two transversals $t_{i j k l}, t_{i j k l}^{\prime}$, the quartics $q_{i j m} q_{k l n}, q_{i k m} q_{j l n}$ formed by the products of quadrics through the lines $\{i, j, m\}$ and $\{k, l, n\}$, and $\{i, k, m\}$ and $\{j, l, n\}$, respectively, satisfy the following quadratic relation:

$$
\begin{aligned}
& {[i k][j l]\left(q_{i j m} q_{k l n}\right)^{2}} \\
& \quad+([i l][j k]-[i j][k l]-[i k][j l]) q_{i j m} q_{k l n} q_{i k m} q_{j l n}+[i j][k l]\left(q_{i k m} q_{j l n}\right)^{2}=0
\end{aligned}
$$

Proof of Lemma 2.3. The proof here rests entirely on straightening laws. Fix a transversal $t_{i j k l}$, and let the point of intersection of $t_{i j k l}$ with the lines $l_{i}, l_{j}, l_{k}, l_{l}$ be given by the points $x_{1}, x_{3}, x_{5}, x_{7}$, respectively. Similarly, denote the points of intersection with the other transversal by the points $x_{2}, x_{4}, x_{6}, x_{8}$. Finally, choose any points $x_{9}, x_{10}$ on $l_{m}$ and $x_{11}, x_{12}$ on $l_{n}$.

It is easy to check that the quadric $q_{i j m}$ can be given by the equation

$$
\left\langle x_{1} x_{2} x_{4} x\right\rangle\left\langle x_{3} x_{9} x_{10} x\right\rangle-\left\langle x_{1} x_{2} x_{3} x\right\rangle\left\langle x_{4} x_{9} x_{10} x\right\rangle,
$$

where $x$ is an arbitrary point in $\mathbf{P}^{3}$, and the brackets represent determinants of the $4 \times 4$ matrices with rows given by the coordinates of the points listed. Also, it can be checked that the quadrics given as such are the same as those described in Section 2.1, and can therefore be used as before to describe the quartics $t_{i}$. By 
permuting the indices, we obtain similar descriptions of the quadrics through any three lines.

We can now describe the transversal $t_{i j k l}$ by $x_{3}+t x_{5}$, and the other transversal by $x_{4}+t x_{6}$. On $t_{i j k l}$, we get the following relation (now writing $i$ for $x_{i}$ ):

$$
\langle 1256\rangle\langle 3478\rangle q_{i j k} q_{l m n}-\langle 1245\rangle\langle 3678\rangle q_{i l m} q_{j k n}=0,
$$

which comes from straightening the tableaux expressions for the quadrics, substituting $x_{3}+t x_{5}$ for $x$. The other transversal yields the relation

$$
\langle 1256\rangle\langle 3478\rangle q_{i j k} q_{l m n}-\langle 1236\rangle\langle 4578\rangle q_{i l m} q_{j k n}=0,
$$

and therefore from the pair of transversals, we get the equation

$$
\begin{aligned}
& \langle 1256\rangle^{2}\langle 3478\rangle^{2}\left(q_{i j k} q_{l m n}\right)^{2}+\langle 1256\rangle\langle 3478\rangle\langle 1234\rangle\langle 5678\rangle q_{i l m} q_{j k n} \\
& -(\langle 1245\rangle\langle 3678\rangle+\langle 1236\rangle\langle 4578\rangle)\langle 1256\rangle\langle 3478\rangle q_{i j k} q_{l m n} q_{i l m} q_{j k n}=0 .
\end{aligned}
$$

Here, we see that

$$
\langle 1245\rangle\langle 3678\rangle+\langle 1236\rangle\langle 4578\rangle=[i l][j k]-[i j][k l]-[i k][j l],
$$

as follows: among the van der Waerden syzygies for tableaux with four columns, we have

$$
\begin{aligned}
\langle 1234\rangle\langle 5678\rangle & -\langle 1235\rangle\langle 4678\rangle+\langle 1236\rangle\langle 4578\rangle+\langle 1245\rangle\langle 3678\rangle \\
& -\langle 1246\rangle\langle 3578\rangle+\langle 1256\rangle\langle 3478\rangle-\langle 1278\rangle\langle 3456\rangle=0 .
\end{aligned}
$$

However, the terms $\langle 1235\rangle\langle 4678\rangle$ and $\langle 1246\rangle\langle 3578\rangle$ vanish, since $\left\{x_{1}, x_{3}, x_{5}, x_{7}\right\}$ are collinear and $\left\{x_{2}, x_{4}, x_{6}, x_{8}\right\}$ are collinear. We are then left with the following relation:

$$
\begin{aligned}
\langle 1245\rangle\langle 3678\rangle+\langle 1236\rangle\langle 4578\rangle & =\langle 1278\rangle\langle 3456\rangle-\langle 1234\rangle\langle 5678\rangle-\langle 1256\rangle\langle 3478\rangle \\
& =[i l][j k]-[i j][k l]-[i k][j l],
\end{aligned}
$$

as desired. The redundant term $\langle 1256\rangle\langle 3478\rangle$ can then be cancelled from equation (2.3), and we are left with the relation stated in the lemma. As it is given in terms of invariants of the lines, it is independent of the points chosen. This proves the lemma.

Recall the syzygies (2.1). As shown before, these syzygies correspond to syzygyies on the quartics $q_{i j k} q_{l m n}$. We can therefore use the information here to compute corresponding relations on the coefficients of the quartics $t_{i}$ which will ensure that the quadric $Q$ vanishes on all transversals. To illustrate this, consider the transversals $t_{1234}, t_{1234}^{\prime}$. Here, $t_{0}=t_{1}=t_{3}=0$, and the quadric $Q$ reduces to

$$
c_{2} t_{2}^{2}+c_{24} t_{2} t_{4}+c_{4} t_{4}^{2}=0 \text {. }
$$

From the lemma, we know

$$
[13][24] t_{2}^{2}+([14][23]-[12][34]-[13][24]) t_{2} t_{4}+[12][34] t_{4}^{2}=0
$$

must hold on the transversals, and so we must have [12][34] $c_{2}=[13][24] c_{4}$ and $([14][23]-[12][34]-[13][24]) c_{2}=[13][24] c_{24}$ so that the preimage of $Q$ in $\mathbf{P}^{3}$ will vanish on the transversals, as desired. We thus obtain two linear relations on the coefficients $c_{*}$ of the quadric $Q$ from each pair of transversals. Although there are then 30 relations and 15 coefficients, there is a unique solution up to proportionality, which is the quadric $Q$.

We can now see that the equation $Q=\lambda h$ must hold in $\mathbf{P}^{3}$ for some $\lambda$. Notice also that, due to the nature of the expressions for $Q$ and $h$ as products of tableaux, 
we must be able to express $\lambda$ as a polynomial in the invariants $q_{i}$ and $D$ with integer coefficients. However, $\lambda$ must be invariant under even permutations of the six lines, and it must change sign when an odd permutation is applied. We thus conclude that $\lambda$ must be a scalar multiple of the invariant $D$. Computations in Mathematica confirm that $\lambda$ is indeed equal to the invariant $D$, where the lines are written in generic coordinates and expanded. These computations were also confirmed by M. Hirsch and R. M. Williams in work supported by the Office of Naval Research Gr.

As shown in [DO], Igusa's quartic $I_{4}$ satisfies the relation

$$
I_{4}=h^{2}
$$

in $\mathbf{P}^{3}$. We can then derive the equation

$$
Q^{2}=D^{2} I_{4}
$$

viewing $Q, I_{4}$ as functions on $\mathbf{P}^{3}$. We can now state the following theorem:

Theorem 2.4. The general equation in $\mathbf{P}^{4}$ which relates the image $K_{4}$ of $\mathbf{P}^{3}$ under $\phi$ to $Q$ and to the Igusa quartic $I_{4}$ is given by

$$
K_{4}+Q^{2}=D^{2} I_{4}
$$

Of particular interest is the following corollary:

Corollary 2.5. For any fixed set of six lines $\left\{l_{i}\right\}$ in $\mathbf{P}^{3}$, the image locus of $\mathbf{P}^{3}$ under $\pi$ is a three-dimensional subvariety of $\mathbf{P}_{2}^{6}$. The sextuples of lines in $\mathbf{P}^{2}$ associated to those in the image of $\pi$ form a dual subvariety in $\mathbf{P}_{2}^{6}$, and this is the image locus of $\mathbf{P}^{3}$ under the map $\pi^{\prime}$ given by the dual lines $\left\{l_{i}^{\prime}\right\}$ in $\mathbf{P}^{3}$.

Recall from Section 1.1 that the moduli space of sextuples of lines in $\mathbf{P}^{2}$ is given in $\mathbf{P}(1,1,1,1,2)$ with coordinates $t_{0}, \ldots, t_{5}$ by the equation

$$
t_{5}^{2}=I_{4}
$$

where $I_{4}$ is Igusa's quartic. Notice that the value of the invariant $t_{5}$ is given as a function of the point of projection from $\mathbf{P}^{3}$ by $h$, just as the $t_{i}$ for $0 \leq i \leq 4$ are regarded here as functions on $\mathbf{P}^{3}$.

By equation (2.2), we see that the image of $\pi$ in $\mathbf{P}(1,1,1,1,2)$ is given by the equations (2.4) and

$$
Q=D t_{5},
$$

where $Q$ is a function of $t_{0}, \ldots, t_{4}$. Notice that, since the dual set of lines has the same invariants $[i j]$, the quadric $Q$ will be the same for the lines and dual lines. However, the invariant $D^{\prime}$ of the dual lines will satisfy $D^{\prime}=-D$. Therefore, the image locus of $\pi^{\prime}$ is given by the equation

$$
Q=-D t_{5},
$$

and is the variety of sextuples of lines which are associated to the sextuples of lines in the image of $\pi$.

This gives a nice interpretation of the commutative diagram in Figure 1: the map $\phi$ is $1: 1$ in the general case, the map $\psi$ is $2: 1$, and the map $\pi$ has a corresponding map $\pi^{\prime}$ which maps to the dual of the image of $\pi$ in $\mathbf{P}_{2}^{6}$.

The following corollary generalizes our result to sets of $m$ lines, $m>6$ : 
Corollary 2.6. For any $m$ lines, $m \geq 6$, such that some subset of six lines is in general position and does not belong to a linear complex, the point of projection can be reconstructed in general from the configuration of image lines.

This follows from the fact that we can construct the map $\phi$ as above for the six general lines, and the map $\phi$ is 1:1. We can thus reconstruct the point of projection exactly when the point does not lie on Hierhölzer's surface.

2.3. Results of Todd. It should be noted at this point that some of the above results were obtained by Todd $[\mathrm{T}$ ] around 1930. Among Todd's results is the following observation: the quartic $K_{4}$ has in general 36 nodes, 30 of which are the images of the transversals. The remaining six nodes are the images of the six cubic curves which have as secants the six lines $l_{i}$. It can be proven via Wakeford's transformation (see [SR], p. 186) that there are in general six cubic curves which have the lines $l_{i}$ as secants, and these are easily shown to blow down to singular points on $K_{4}$.

Todd gives the following result for the case in which the six lines $l_{i}$ are the six lines of a linear complex, i.e. $D=0$ :

Theorem 2.7. Given six lines $l_{1}, \ldots l_{6}$ which lie in a linear complex, the corresponding surface $\mathrm{O}_{8}$ maps to an octic surface in $\mathbf{P}^{4}$ which has thirty-six nodes.

Todd shows in his paper that when the six lines $l_{i}$ are linearly dependent in the Plücker space, the six twisted cubics which have the six lines as secants then lie on Hierhölzer's surface. The image of $O_{8}$ under the canonical map then contains nodes corresponding to both the six cubics and the thirty transversals.

It is evident from the equation $K_{4}+Q^{2}=D I_{4}$ that when the six lines $l_{i}$ are linearly dependent in the Plücker space, the corresponding map to $\mathbf{P}^{4}$ has as its image a quadric rather than a quartic threefold in $\mathbf{P}^{4}$. The map from $\mathbf{P}^{3}$ to $\mathbf{P}^{4}$ is then $2: 1$, rather than $1: 1$ as in the general case. Todd describes a Cremona transformation of $\mathbf{P}^{3}$ that is given by surfaces of degree 19 which pass quintuply through the six lines, and which acts by switching points in $\mathbf{P}^{3}$ which are mapped to the same point in $\mathbf{P}^{4}$ under the double cover.

2.4. Hierhölzer's Surface from a Double-Sixer. We will now consider the special case of Hierhölzer's surface in which the six lines $\left\{l_{1}, \ldots, l_{6}\right\}$ are the six lines of a double-sixer on a cubic surface.

In this case, it can be quickly checked that the quartics $\left\{t_{i}\right\}$ are the same as the quartics given by the dual lines of the double sixer, up to a change of sign. In this case, then, both the original lines and the dual lines are blown up in the map given by the canonical divisor. We can show that the exceptional divisors corresponding to the lines and dual lines map to conics on Hierhölzer's surface, using the following corollary to Theorem 2.4:

Corollary 2.8. The equation in Theorem 2.4 also holds for six nonintersecting lines on a nonsingular cubic surface, with coefficients of the quadric $Q$ given as before in Theorem 2.2.

This can be verified directly via computations in Mathematica. Here, the additional structure of the double sixer limits the number of parameters needed to describe the configuration of lines, and therefore the calculations are greatly simplified. 
Now, without loss of generality, we can assume that line $l_{1}$ has equation $x_{0}=$ $x_{1}=0$ in $\mathbf{P}^{3}$. We then change to the following coordinate system to describe the linear system of quartics which vanish on all six lines:

$$
\left\{h_{0}, h_{1}, h_{2}, h_{3}, h_{4}\right\}=\left\{x_{0} F_{3}, x_{1} F_{3}, x_{2} F_{3}, x_{3} F_{3}, L\right\},
$$

where $F_{3}$ is the cubic surface, $x_{i}$ are coordinates on $\mathbf{P}^{3}$, and $L$ is the quartic $q_{123} q_{456}$. It is then possible to find a projective automorphism of $\mathbf{P}^{4}$ which will change from the original coordinates to the new ones, and to use this to determine the equation $Q^{\prime}$ of the quadric $Q$ in the new variables. Notice that the equation of the quartic $K_{4}$ can be given in the new variables by the equation

$$
L\left(h_{0}, h_{1}, h_{2}, h_{3}\right)-h_{4} F_{3}\left(h_{0}, h_{1}, h_{2}, h_{3}\right)=0 .
$$

To determine the image of the exceptional divisor after blowing up the line $l_{1}$, we observe that the coordinates $h_{0}, h_{1}$ vanish with multiplicity two on $l_{1}$ and the remaining three coordinates vanish with multiplicity one. We thus conclude that the image of the exceptional divisor of $l_{1}$ must be contained in the conic intersection of the plane $h_{0}=h_{1}=0$ and the quadric $Q^{\prime}$. We then conclude by symmetry that the images of the exceptional divisors corresponding to both the lines and dual lines map to conics on the canonical model of $O_{8}$.

There are 30 intersection points of the lines and dual lines in $\mathbf{P}^{3}$. These correspond to the 30 singular points on the canonical model, as follows: the lines and dual lines blow down to conics on the canonical model, and the conic images of a line and its dual line intersect in a single point. Just as the singular points occur in pairs, the intersection points in $\mathbf{P}^{3}$ occur in corresponding pairs: for example, there are points $l_{1} \cap l_{2}^{\prime}$ and $l_{1}^{\prime} \cap l_{2}$ in $\mathbf{P}^{3}$; these correspond to the nodes on the canonical model which represent configurations in $\mathbf{P}_{2}^{6}$ wherein the images of the lines $l_{3}, l_{4}, l_{5}$, and $l_{6}$ intersect in a single point.

Each of the above conics necessarily lies in a plane. These twelve planes intersect in a single point, which does not lie on the canonical model of the surface. This point is the image of the cubic surface on which the twelve lines and transversals lie. The dual of this point is a hyperplane in $\mathbf{P}^{4}$. Projection of the canonical model of the surface from the point to the hyperplane yields an octic surface in $\mathbf{P}^{3}$ with twelve double lines. We can see that this is isomorphic in $\mathbf{P}^{3}$ to the original surface, as follows: in our new coordinates, the surface must then blow down to the point $(0,0,0,0,1)$, as the quartics $h_{0}, h_{1}, h_{2}, h_{3}$ all vanish on the cubic surface. Projection from this point to the plane $h_{4}=0$ returns us to the original surface.

\section{REFERENCES}

[Ba] H. Baker. Principles of Geometry, Vol. 3. Cambridge Univ. Press, 1927.

[Ca] A. Cayley. On the surfaces each the locus of the vertex of a cone which passes through $m$ given points and touches $6-m$ given lines. Proc. London Math. Soc., 4:11-47, 1872.

[Co] A. Coble. Algebraic Geometry and Theta Functions. Amer. Math. Soc., 1929.

[DO] I. Dolgachev and D. Ortland. Point sets in projective space and theta functions. Asterisque, Volume 165. Soc. Math. France, 1989. MR 90i:14009

[D] I. Dolgachev. Introduction to geometric invariant theory. Lecture Notes Series, Number 25. Seoul National University, 1994. MR 96a:14019

[GH] P. Griffiths and J. Harris. Principles of Algebraic Geometry. Wiley, 1978. MR 80b:14001

[Gr] F. Grosshans, R. Gleeson, M. Hirsch, R. M. Williams. Object-image equations for x points and 6 - x lines. Preprint, 1998.

[Ha] R. Hartshorne. Algebraic Geometry. Springer-Verlag, 1977. MR 57:3116

[Hz] V. Hierhölzer. Ueber Kegelschnitte im Raume. Math. Ann., 2:563 -585, 1870. 
[Hg] R. Huang. Combinatorial Methods in Invariant Theory. PhD thesis, Massachusetts Institute of Technology, 1993.

[Ht] B. Hunt. The Geometry of Some Special Arithmetic Quotients. Springer-Verlag, 1996. MR 98c:14033

[Ht2] http://www.mathematik.uni-kl.de/ ${ }^{\sim}$ wwwagag/E/Galerie.html

[K] M. Kapranov. Veronese Curves and Grothendieck-Knudsen Moduli Space. J. Alg. Geom., 2:239-262, 1993. MR 94a:14024

$[\mathrm{M}] \quad$ D. Mumford. Geometric Invariant Theory. Springer-Verlag, 1965. MR 35:5451

[Sc] I. Schur. Uber die durch collineare grundgebilde erzeugten curven und flachen. Math. Ann., $18: 1-32,1881$

[SR] J. Semple and S. Roth. Introduction to Algebraic Geometry. Clarendon Press, 1949. MR 11:535d

[Stu] R. Sturm. Die Gebilde ersten und zweiten Grades der liniengeometrie in synthetischer Behandlung, Vol. 1. B. G. Teubner, 1892.

[Sf] B. Sturmfels. Algorithms in Invariant Theory. Springer-Verlag, 1993. MR 94m:13004

[T] J. Todd. Configurations defined by six lines. Proc. Cambridge Phil. Soc., 29:52-68, 1932.

[W] H. Weyl. The Classical Groups: Their Invariants and Representations. Princeton University Press, 1946. MR 1:42c

[Z] O. Zariski. Algebraic Surfaces. Springer-Verlag, 1995. MR 96c:14024

Department of Mathematics and Computer Science, Truman State University, Kirksville, Missouri 63501

E-mail address: dvazzana@truman.edu 